\title{
EHMTI-0338. The enzymes phosphodiesterase 3 and 5 express activity in the trigeminal ganglion and co-localize with calcitonin gene-related peptide
}

\author{
JC Nordgaard ${ }^{1 *}$, LS Kruse ${ }^{2}$, M Moeller $^{3},{\text { C } \text { Kruuse }^{4}}^{4}$ \\ From 4th European Headache and Migraine Trust International Congress: EHMTIC 2014 \\ Copenhagen, Denmark. 18-21 September 2014
}

\section{Introduction}

The neuropathology leading to migraine pain has centered on an either a vascular or neuronal origin. Sildenafil, a specific inhibitor of phosphodiesterase 5 (PDE5), was the first compound to induce migraine-like headache in a human headache model without a concomitant artery dilatation.

\section{Aim}

In this study we investigated the presence and activity of PDE3 and PDE5 in a key component of the neuronal pathway, the rat trigeminal ganglion. We further addressed a possible cross-talk with a key molecule in migraine pain signaling, calcitonin gene related peptide (CGRP), by investigating cellular co-localization.

\section{Methods}

Analyzes were performed on isolated rat trigeminal ganglion. Localization was done by immunohistochemistry, in situ hybridization, and western blots. Enzymatic assays for cAMP and cGMP hydrolysis were done using scintillation proximity assay.

\section{Results}

We show that PDE3 and PDE5 are present and express activity in the trigeminal ganglia. PDE3 and PDE5 were observed in the majority of neurons in the ganglion. However, only a subset of these PDE3 and PDE5 positive cells contained CGRP.

\section{Conclusions}

Hydrolysis of cyclic AMP was in contrast to cGMP, influenced by both sildenafil and cilostazol, suggesting sildenafil, cilostazol and CGRP may work through a common cAMP pathway resulting in migraine pain.

No conflict of interest.

\section{Authors' details}

${ }^{1}$ University of Copenhagen, Glostrup Hospital Lundbeck Foundation Center for Vascular research PDE group Glostrup Research Park, Glostrup, Denmark. ${ }^{2}$ University of Copenhagen, Glostrup Hospital Glostrup Research Park, Glostrup, Denmark. ${ }^{3}$ Dept Neuroscience and Pharmacology, Panum Institute Univeristy of Copenhagen, Copenhagen, Denmark. ${ }^{4}$ University of Copenhagen, Herlev Hospital Dept Neurology and Lundbeck FOundation Center for Vascular Research PDE group, Herlev, Denmark.

Published: 18 September 2014

doi:10.1186/1129-2377-15-S1-F14

Cite this article as: Nordgaard et al:: EHMTI-0338. The enzymes phosphodiesterase 3 and 5 express activity in the trigeminal ganglion and co-localize with calcitonin gene-related peptide. The Journal of Headache and Pain 2014 15(Suppl 1):F14. 